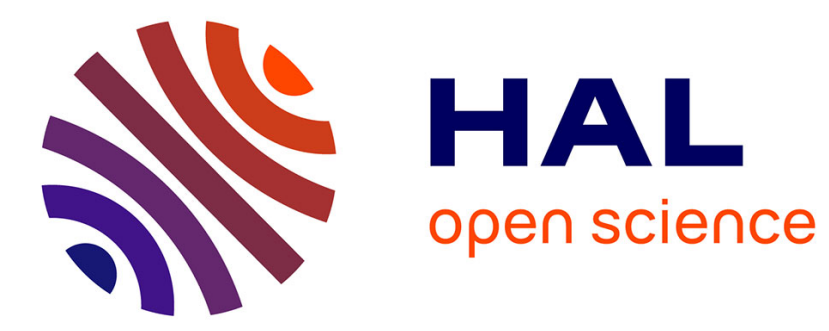

\title{
On Solutions of a Non linear Wave Equation Derived From Brain Activity Modeling
}

\author{
Mouhamad Jradeh, Maïtine Bergounioux
}

\section{To cite this version:}

Mouhamad Jradeh, Maïtine Bergounioux. On Solutions of a Non linear Wave Equation Derived From Brain Activity Modeling. 2008. hal-00126996v3

\section{HAL Id: hal-00126996 https://hal.science/hal-00126996v3}

Preprint submitted on 19 Apr 2008

HAL is a multi-disciplinary open access archive for the deposit and dissemination of scientific research documents, whether they are published or not. The documents may come from teaching and research institutions in France or abroad, or from public or private research centers.
L'archive ouverte pluridisciplinaire HAL, est destinée au dépôt et à la diffusion de documents scientifiques de niveau recherche, publiés ou non, émanant des établissements d'enseignement et de recherche français ou étrangers, des laboratoires publics ou privés. 


\title{
On Solutions of a Non linear Wave Equation Derived From Brain Activity Modeling
}

\author{
Mouhamad Jradeh and Maïtine Bergounioux \\ Laboratoire MAPMO- UMR 6628 - Fédération Denis Poisson - Université \\ d'Orléans - BP 6759 - 45067 Orléans cedex 02, France
}

\begin{abstract}
The paper deals with a boundary value problem governed by a damped non-linear wave equation. It generalizes the equation modelling cerebral activity that has been proposed by Jirsa and Haken [9]. Using a Galerkin approximation scheme, we prove existence of global solutions. In the 1-D case, we show the continuous dependence of solutions on the initial data and derive uniqueness.
\end{abstract}

Key words: Neural fields, wave equation, Galerkin method, boundary value problem

1991 MSC: : 35L05, 35Q80

\section{Introduction}

Non-invasive techniques such as functional Magnetic Resonance Imaging, ElectroEncephaloGraphy (EEG) and MagnetoEncephaloGraphy (MEG) provide entry points to human brain dynamics for clinical purposes, as well as the study of human behavior and cognition. Each of these observation technologies provides spatiotemporal information about the ongoing neural activity in the cortex. Unfortunately measures are generally noisy, and it is difficult to identify the equation which governs the dynamics of neural activity. Several physicists $[23,16,9,7]$ have formulated continuous models called neural fields to predict neural activity using brain anatomy.

Jirsa and Haken's model [9], which we will analyze in section 4 of this paper, generalizes the models $[23,16]$ and leads to the following evolution problem that we investigate in this work:

Email addresses: mouhamad.jradeh@univ-orleans.fr (Mouhamad Jradeh ), maitine. bergounioux@univ-orleans.fr (Maïtine Bergounioux). 


$$
\begin{aligned}
u_{t t}-\alpha \Delta u=a(u, p) u_{t}+b\left(u, p, p_{t}\right), \quad \text { in }[0,+\infty) \times \Omega & \\
u(0, \cdot)=u_{0} & u_{t}(0, \cdot)=u_{1} \cdot \\
u=0, \quad \text { on }[0,+\infty) \times \partial \Omega &
\end{aligned}
$$

where $\Omega$ is an open bounded domain in $\mathbb{R}^{n}(n \leq 4)$ with sufficiently smooth boundary $\partial \Omega$. The notation $u_{t}$ (respectively $u_{t t}$ ) stands for the first (respectively second) partial derivative of $u$ with respect to time variable $t, \Delta$ is the Laplace operator and $p$ is a smooth given function modelling the external stimulus (input). For simplicity, we assume that $\alpha=1$.

Although equation (1.1) has a simple form, the existence and uniqueness of the solution is (to our knowledge) still an open problem, if we do not assume additional conditions on $a, b$ or $u_{0}, u_{1}$. In addition, the existence of a global solution is not always assured (see for example $[5,28,25]$ ).

Equation (1.1) belongs to a class of non-linear damped wave equations that have been widely studied by many mathematicians and engineers. Existence and uniqueness under conditions (1.2) and (1.3) has been considered using various methods such as semi-group theory $[17,14,27,3]$ and fixed point methods $[10,2]$. In $[6,4,18,19,26]$, the authors prove existence and uniqueness of global solutions under conditions (1.2) and (1.3) using Galerkin methods.

Zhou [28] has studied a particular case of equation (1.1), without external input $p$. The function $a$ is assumed to be constant and $b$ is defined by

$$
b(u)=|u|^{m-1} u
$$

with condition (1.2) and $\Omega=\mathbb{R}^{n}$; he proved that, if $1<m<\frac{n+2}{n}$, the solution blows up in finite time. Similarly, authors in $[17,24,28,29,11,12,21,22,5,15,25]$ discussed the case when the solution blows up in finite time.

The closest equation to the one we investigate here has been studied by Zhijian [26], who considered:

$$
\begin{aligned}
u_{t t}-\Delta u-\Delta u_{t}= & \sum_{i=1}^{n} \frac{\partial}{\partial x_{i}}\left(\sigma_{i}\left(u_{x_{i}}\right)+\beta_{i}\left(u_{x_{i t}}\right)\right) \\
& +F\left(u, u_{t}, \nabla u, \nabla u_{t}\right) \quad \text { on }[0, \infty) \times \Omega
\end{aligned}
$$

with initial and boundary conditions (1.2) and (1.3). He proved that this problem has a unique classical solution, assuming the following about the initial data:

$$
\begin{aligned}
& \left\|\sum_{i=1}^{n} \frac{\partial}{\partial x_{i}}\left(\sigma_{i}\left(u_{x_{i}}(0)\right)+\beta_{i}\left(v_{x_{i}}(0)\right)\right)+F(u(0), v(0), \nabla u(0), \nabla v(0))\right\|_{H^{k}}<\delta \\
& \|v(0)\|_{H^{k+1}}^{2}+\|v(0)\|_{H^{k}}+\frac{3}{2}\|u(0)\|_{H^{k}}+<u(0), v(0)>_{H^{k}} \quad<\gamma
\end{aligned}
$$

where $\delta$ and $\gamma$ are two real numbers. In the present section, $H^{k}$ stands for the usual Sobolev space and $\|\cdot\|_{H^{k}}$ denotes the $H^{k}$-norm. This notation will 
be changed in the sequel, where we shall use $H^{1}$ and $H^{2}$. In addition, it was supposed that

$$
\left|D^{\beta} F\right| \leq B\left(\left|\lambda_{1}\right|^{\gamma_{1}}+\left|\lambda_{2}\right|^{\gamma_{2}}+\left|\tilde{\lambda_{1}}\right|^{\gamma_{3}}+\left|\tilde{\lambda}_{2}\right|^{\gamma_{4}}+1\right) \text { and } F(0)=0
$$

The RHS of (1.1) is a particular case of the RHS in (1.4): it involves an additional (parameter) function $p$ that we call external input. This function physically describes a signal generated at the brain surface and transmitted to the neocortex. We have to mention here that the term $\Delta u_{t}$ on the LHS of equation (1.4) is crucial in the proof estimates: the solution is bounded with respect to the $H^{2}$ norm.

Now, the question is: if the initial data does not satisfy (1.5), (1.6) or (1.7) and if no "regularizing" term $\Delta u_{t}$ appears, does problem (1.1)-(1.3) admit a global solution? If yes, is the problem mathematically well posed: is the solution unique and continuous with respect to initial data?

In this paper, we prove that under "realistic" assumptions, problem (1.1)(1.3) has global weak solutions. Moreover, if the problem is a unidimensional one, the solutions depend continuously on the intial data; therefore we get uniqueness. Required assumptions are motivated by the underlying physical cerebral activity model that leads to a particular case of equation (1.1).

The outline of the paper is the following: existence results are stated in Section 2. Uniqueness and stability are discussed in Section 3. Finally, in Section 4, we present and analyze the cerebral activity model and we apply the general result.

\section{Existence of weak global solutions}

We let $\|\cdot\|$ denote the $L^{2}(\Omega)$ norm, $\|\cdot\|_{\infty}$ the $L^{\infty}$-norm, $\|\cdot\|_{1}$ the $H^{1}(\Omega)$ norm, and $(\cdot, \cdot)$ the $L^{2}$-inner product.

Definition 2.1 Let $\left(u_{0}, u_{1}\right) \in H_{0}^{1}(\Omega) \times H_{0}^{1}(\Omega)$, and $T>0$ be given. We shall say that $u$ is a weak solution to problem (1.1)-(1.3) if

$$
u \in L^{2}\left([0, T), H_{0}^{1}(\Omega)\right), u_{t} \in L^{2}\left([0, T), H^{1}(\Omega)\right), u_{t t} \in L^{2}\left([0, T), L^{2}(\Omega)\right)
$$

and for almost every $t \geq 0, \forall \omega \in H_{0}^{1}(\Omega)$

$$
\left(u_{t t}(t), \omega\right)+(\nabla u(t), \nabla \omega)=\left(a(u, p)(t) u_{t}(t)+b\left(u, p, p_{t}\right)(t), \omega\right)
$$

and

$$
u(0, \cdot)=u_{0}, \quad u_{t}(0, \cdot)=u_{1}
$$


From now on, we do not indicate the dependence on $t$ in order to make the paper more readable. First we give an existence result of weak solutions to problem (1.1)-(1.3).

Theorem 2.1 Assume that for some $T>0$, $\left(A_{1}\right) a \in C^{1}\left(\mathbb{R}^{2}, \mathbb{R}\right)$ and $b \in C^{1}\left(\mathbb{R}^{3}, \mathbb{R}\right)$, where $a, \nabla a$ and $\nabla b$ are (uniformly) bounded functions.

$\left(A_{2}\right)|b(u, p, q)(t, x)| \leq C_{1}|u(t, x)|+C_{2} \quad$ a.e. on $[0, T) \times \Omega$, where $C_{1}$ and $C_{2}$ are nonnegative constants.

$\left(A_{3}\right) p \in H^{2}\left([0, T), L^{\infty}(\Omega)\right)$.

$\left(A_{4}\right)\left(u_{0}, u_{1}\right) \in H_{0}^{1}(\Omega) \times H_{0}^{1}(\Omega)$

Then the problem (1.1)-(1.3) admits (at least) a weak solution $u$ on $[0, T)$ and

$$
u \in H^{2}\left([0, T), L^{2}(\Omega)\right) \cap H^{1}\left([0, T), H_{0}^{1}(\Omega)\right) .
$$

Proof - We need several steps to prove this result. We use the Galerkin method to construct a solution. Let $\left\{\lambda_{k}\right\}_{k=1}^{\infty}$ be a sequence of eigenvalues of $-\Delta$ in $\Omega$. Let $\left\{\omega_{k}\right\}_{k=1}^{\infty} \in H_{0}^{1}(\Omega) \cap H^{2}(\Omega)$ be the associated eigenfunctions such that $\left\{\omega_{k}\right\}_{k=1}^{\infty}$ is a complete orthonormal system of $L^{2}(\Omega)$. We construct approximate solutions $u^{n}$ as

$$
u^{n}(t)=\sum_{k=1}^{n} d^{n, k}(t) \omega_{k}
$$

where the functions $d^{n, k}$ are determined by the system of ordinary differential equations

$$
\begin{aligned}
\left(u_{t t}^{n}, \omega_{k}\right)+\left(\nabla u^{n}, \nabla \omega_{k}\right) & =\left(a\left(u^{n}, p\right) u_{t}^{n}+b\left(u^{n}, p, p_{t}\right), \omega_{k}\right) \quad k=1, \ldots n . \\
d^{n, k}(0) & =u_{0}^{k}, \quad d_{t}^{n, k}(0)=u_{1}^{k}
\end{aligned}
$$

with $u_{0}^{k}, u_{1}^{k} \in \mathcal{D}(\Omega)$, such that $\left(u_{0}^{k}, u_{1}^{k}\right) \rightarrow\left(u_{0}, u_{1}\right)$ in $H_{0}^{1}(\Omega) \times H_{0}^{1}(\Omega)$. Here $\mathcal{D}(\Omega)$ denotes the space of $\mathcal{C}^{\infty}$ functions with compact support in $\Omega$.

Now, (2.3) and (2.4) are equivalent to:

$$
\begin{gathered}
d_{t t}^{n, k}+\lambda_{k} d^{n, k}=f\left(d^{n, k}, d_{t}^{n, k}, t\right) \quad k=1, \ldots n \\
d^{n, k}(0)=u_{0}^{k}, \quad d_{t}^{n, k}(0)=u_{1}^{n}
\end{gathered}
$$

where

$$
f\left(d^{n, k}, d_{t}^{n, k}, t\right):=\left(a\left(u^{n}, p\right) u_{t}^{n}+b\left(u^{n}, p, p_{t}\right), \omega_{k}\right) \quad \text { a.e. } \quad \mathrm{t} \geq 0
$$

For every $n \geq 1,(2.5),(2.6)$ is a second-order $n \times n$ Cauchy system of differential equations with continuous nonlinearities. So, it follows from the CauchyPeano Theorem [20] that the system has at least one solution $d^{n, k}$ defined on 
$\left[0, T_{n}\right]$ for some $T_{n}>0$. Moreover, for every $k$ such that $1 \leq k \leq n$, we get $d^{n, k} \in C^{2}\left(\left[0, T_{n}\right]\right)$.

Lemma 2.1 The functions $\left(u_{n}\right)$ satisfy

$$
\left\|u_{t}^{n}(t)\right\|+\left\|u^{n}(t)\right\|_{1} \leq M(T)
$$

where $M(T)$ only depends on $T$. In particular, $u^{n}(t)$ can be extended to $[0, T)$.

Proof - We multiply equation (2.3) by $d_{t}^{n, k}$ and sum over $\mathrm{k}=1, \ldots, \mathrm{n}$. By assumptions $A_{1}$ and $A_{2}$ we obtain, for every $t \in[0, T)$,

$$
\begin{aligned}
\left(u_{t t}^{n}, u_{t}^{n}\right)+\left(\nabla u^{n}, \nabla u_{t}^{n}\right) & =\left(a\left(u^{n}, p\right) u_{t}^{n}+b\left(u^{n}, p, p_{t}\right), u_{t}^{n}\right) \\
& \leq C\left(\left|u_{t}^{n}\right|+C_{1}\left|u^{n}\right|+C_{2},\left|u_{t}^{n}\right|\right) .
\end{aligned}
$$

Using the Cauchy-Schwartz inequality, we have

$$
\begin{aligned}
\frac{d}{d t}\left(\left\|u_{t}^{n}(t)\right\|^{2}+\left\|\nabla u^{n}(t)\right\|^{2}\right) & \leq C\left(\left\|u_{t}^{n}(t)\right\|^{2}+\left\|u^{n}(t)\right\|\left\|u_{t}^{n}(t)\right\|+\left\|u_{t}^{n}(t)\right\|\right) \\
& \leq C\left(\left\|u_{t}^{n}(t)\right\|^{2}+\left\|u^{n}(t)\right\|^{2}+\left\|u_{t}^{n}(t)\right\|^{2}+\left\|u_{t}^{n}(t)\right\|^{2}+1\right) \\
& \leq C\left(\left\|u_{t}^{n}(t)\right\|^{2}+\left\|u^{n}(t)\right\|^{2}+1\right) \\
& \leq C\left(\left\|u_{t}^{n}(t)\right\|^{2}+\left\|\nabla u^{n}(t)\right\|^{2}+1\right)
\end{aligned}
$$

by the Poincaré inequality, where $C$ denotes (here and in the sequel) a generic constant. Applying the Gronwall inequality yields

$$
\left\|u_{t}^{n}(t)\right\|+\left\|\nabla u^{n}(t)\right\| \leq M(T) ;
$$

then, by the Poincaré inequality,

$$
\left\|u_{t}^{n}(t)\right\|+\left\|u^{n}(t)\right\|_{1} \leq M(T)
$$

and

$$
\begin{aligned}
\left|f\left(d^{n, k}, d_{t}^{n, k}, t\right)\right| & =\left|\left(a\left(u^{n}, p\right) u_{t}^{n}+b\left(u^{n}, p, p_{t}\right), \omega_{k}\right)\right| \quad \text { a.e. } \mathrm{t} \geq 0 \\
& \leq C\left(\left\|u_{t}^{n}(t)\right\|^{2}+\left\|u^{n}(t)\right\|^{2}\right) \leq C .
\end{aligned}
$$

So $f\left(d^{n, k}, d_{t}^{n, k}, \cdot\right)$ is a bounded function, and the solution can be extended to $[0, T)[20]$.

Lemma 2.2 The sequence of approximated solutions $u^{n}$ satisfies the following:

(1) $u^{n}$ is bounded in $L^{\infty}\left([0, T), H_{0}^{1}(\Omega)\right.$ ) (and in $L^{2}\left([0, T), H_{0}^{1}(\Omega)\right.$ ).

(2) $u_{t}^{n}$ is bounded in $L^{\infty}\left([0, T), H_{0}^{1}(\Omega)\right.$ ) (and in $L^{2}\left([0, T), H_{0}^{1}(\Omega)\right.$ ). 
(3) $u_{t t}^{n}$ is bounded in $L^{\infty}\left([0, T), L^{2}(\Omega)\right)$ (and in $L^{2}\left([0, T), L^{2}(\Omega)\right)$.

Proof - The first item is an immediate consequence of Lemma 2.1. Differentiating (2.3) with respect to $t$ gives, for $k=1, \ldots n$,

$$
\begin{aligned}
\left(u_{t t t}^{n}, \omega_{k}\right)+\left(\nabla u_{t}^{n}, \nabla \omega_{k}\right) & =\left(a_{u}\left(u^{n}, p\right)\left(u_{t}^{n}\right)^{2}+a_{p}\left(u^{n}, p\right) p_{t} u_{t}^{n}\right. \\
& +a\left(u^{n}, p\right) u_{t t}^{n}+b_{u}\left(u^{n}, p, p_{t}\right) u_{t}^{n}+b_{p}\left(u^{n}, p, p_{t}\right) p_{t} \\
& \left.+b_{q}\left(u^{n}, p, p_{t}\right) p_{t t}, \omega_{k}\right) \quad k=1, \ldots n \\
& \leq C\left(\left(u_{t}^{n}\right)^{2}+\left|u_{t}^{n}\right|+\left|u_{t t}^{n}\right|+\left|u_{t}^{n}\right|+M,\left|\omega_{k}\right|\right),
\end{aligned}
$$

with assumptions $A_{1}, A_{2}$ and $A_{3}$. Here, $a_{u}$ and $a_{p}$ denote the partial derivatives of $a:(u, p) \mapsto a(u, p)$. Similarly, $b_{u}, b_{p}$ and $b_{q}$ are the partial derivatives of $b:(u, p, q) \mapsto b(u, p, q)$.

The method is similar to the one used in the previous Lemma. Replace now $\omega_{k}$ by $u_{t t}^{n}(t)$ :

$$
\begin{aligned}
\frac{d}{d t}\left(\left\|u_{t t}^{n}(t)\right\|^{2}+\left\|\nabla u_{t}^{n}(t)\right\|^{2}\right) & \leq C\left(\left\|\left(u_{t}^{n}(t)\right)^{2}\right\|+\left\|u_{t}^{n}(t)\right\|+\left\|u_{t t}^{n}(t)\right\|+M\right)\left\|u_{t t}^{n}(t)\right\| \\
& \leq C\left(\left\|\left(u_{t}^{n}(t)\right)^{2}\right\|\left\|u_{t t}^{n}(t)\right\|+\left\|u_{t}^{n}(t)\right\|\left\|u_{t t}^{n}(t)\right\|+\right. \\
& \left.\left\|u_{t t}^{n}(t)\right\|^{2}+\left\|u_{t t}^{n}(t)\right\|\right) \\
& \leq C\left(\left\|\left(u_{t}^{n}(t)\right)^{2}\right\|^{2}+\left\|u_{t t}^{n}(t)\right\|^{2}+\left\|u_{t}^{n}(t)\right\|^{2}+\right. \\
& \left.\left\|u_{t t}^{n}(t)\right\|^{2}+\left\|u_{t t}^{n}(t)\right\|^{2}+\left\|u_{t t}^{n}(t)\right\|^{2}+1\right) \\
& \leq C\left(\left\|\left(u_{t}^{n}(t)\right)^{2}\right\|^{2}+\left\|u_{t t}^{n}(t)\right\|^{2}+\left\|u_{t}^{n}(t)\right\|^{2}+1\right)
\end{aligned}
$$

Using the Sobolev-Poincaré inequality [1],

$$
\left\|u_{t}^{n}(t)\right\|_{L^{4}} \leq C\left\|u_{t}^{n}(t)\right\|_{1}
$$

and the Poincaré inequality, we get

$$
\begin{aligned}
\frac{d}{d t}\left(\left\|u_{t t}^{n}(t)\right\|^{2}+\left\|\nabla u_{t}^{n}(t)\right\|^{2}\right) & \leq C\left(\left\|u_{t t}^{n}(t)\right\|^{2}+\left\|u_{t}^{n}(t)\right\|_{1}^{2}+1\right) \\
& \leq C\left(\left\|u_{t t}^{n}(t)\right\|^{2}+\left\|\nabla u_{t}^{n}(t)\right\|^{2}+1\right)
\end{aligned}
$$

Then by the Gronwall inequality we obtain

$$
\left\|u_{t}^{n}(t)\right\|_{1}+\left\|u_{t t}^{n}(t)\right\| \leq M(T) \quad \text { a.e. } \mathrm{t} \in[0, \mathrm{~T}[,
$$

and the lemma is proved. 
Thanks to estimations (2.9) and (2.10), we may extract subsequences of $\left\{u^{n}\right\}$ (denoted similarly in the sequel) such that

$u^{n} \rightarrow u \quad$ star-weakly in $L^{\infty}\left([0, T), H_{0}^{1}(\Omega)\right)$ and weakly in $L^{2}\left([0, T), H_{0}^{1}(\Omega)\right)$ $u_{t}^{n} \rightarrow u_{t} \quad$ star-weakly in $L^{\infty}\left([0, T), H_{0}^{1}(\Omega)\right)$ and weakly in $L^{2}\left([0, T), H_{0}^{1}(\Omega)\right)$, $u_{t t}^{n} \rightarrow u_{t t} \quad$ star-weakly in $L^{\infty}\left([0, T), L^{2}(\Omega)\right)$ and weakly in $L^{2}\left([0, T), L^{2}(\Omega)\right)$. By classical compactness results [13],

$$
u^{n} \rightarrow u \quad \text { strongly in } \quad L^{2}\left([0, T), L^{2}(\Omega)\right) .
$$

With the above convergence results, we can extract subsequences $\left\{u^{n}\right\}$ such that

$$
\begin{array}{crl}
u^{n}(t, x) \rightarrow u(t, x) & \text { a.e. }(\mathrm{t}, \mathrm{x}) \in[0, \mathrm{~T}) \times \Omega \\
u^{n}(t, x) \leq g(t, x) & \text { a.e. }(\mathrm{t}, \mathrm{x}) \in[0, \mathrm{~T}) \times \Omega \\
u_{t}^{n}(t) \rightarrow u_{t}(t) & \text { weakly in } H_{0}^{1}(\Omega), \quad \text { a.e. } \mathrm{t} \in[0, \mathrm{~T}) .
\end{array}
$$

Now, by continuity of $a$ and $b,(A 1)$, and (2.12), we obtain

$$
\begin{aligned}
a^{4}\left(u^{n}(t, x), p(t, x)\right) & \rightarrow a^{4}(u(t, x), p(t, x)) \\
b\left(u^{n}(t, x), p(t, x), p_{t}(t, x)\right) & \rightarrow b\left(u(t, x), p(t, x), p_{t}(t, x)\right)
\end{aligned}
$$

for almost every $(t, x) \in[0, T) \times \Omega$. Using Lebesgue's theorem, by (2.15) and $\left(A_{1}\right)$ we obtain

$$
a\left(u^{n}(t), p(t)\right) \longrightarrow a(u(t), p(t)) \text { in }^{4}(\Omega) \quad \text { a.e. } \mathrm{t} \in[0, \mathrm{~T})
$$

and by $(2.16),(2.13)$ and $\left(A_{2}\right)$ we obtain

$$
b\left(u^{n}(t), p(t), p_{t}(t)\right) \rightarrow b\left(u(t), p(t), p_{t}(t)\right) \text { in } \mathrm{L}^{2}(\Omega) \quad \text { a.e. } \mathrm{t} \in[0, \mathrm{~T})
$$

On the other hand, for all $v \in L^{2}$, we have

$$
\begin{gathered}
\left|\left(a\left(u^{n}, p\right) u_{t}^{n}-a(u, p) u_{t}, v\right)\right|=\left|\left(a\left(u^{n}, p\right) u_{t}^{n}-a(u, p) u_{t}^{n}+a(u, p) u_{t}^{n}-a(u, p) u_{t}, v\right)\right| \\
\left.\leq \mid\left(a\left(u^{n}, p\right)-a(u, p)\right) u_{t}^{n}, v\right)|+|\left(a(u, p)\left(u_{t}^{n}-u_{t}\right), v\right) \mid \\
\leq\left\|a\left(u^{n}, p\right)-a(u, p)\right\|_{L^{4}}\left\|u_{t}^{n}\right\|_{L^{4}}\|v\|+\left|\left(u_{t}^{n}-u_{t}, a(u, p) v\right)\right| \\
\leq C\left\|a\left(u^{n}, p\right)-a(u, p)\right\|_{L^{4}}\left\|u_{t}^{n}\right\|_{1}\|v\|+\left|\left(u_{t}^{n}-u_{t}, a(u, p) v\right)\right| .
\end{gathered}
$$

Using (2.17), (2.10) and (2.14), we obtain for almost every $t \in[0, T)$

$$
a\left(u^{n}(t), p(t)\right) u_{t}^{n}(t) \rightarrow a(u(t), p(t)) u_{t}(t) \text { weakly in } L^{2}(\Omega) .
$$


Then, using (2.18) and (2.19), and letting $n \rightarrow \infty$ in (2.3), we obtain that the limiting function $u \in H^{2}\left([0, T), L^{2}(\Omega)\right) \cap H^{1}\left([0, T), H_{0}^{1}(\Omega)\right)$ and for almost every $t \in[0, T)$,

$$
\begin{aligned}
\left(u_{t t}, \omega\right)+(\nabla u, \nabla \omega) & =\left(a(u, p) u_{t}+b\left(u, p, p_{t}\right), \omega\right) \quad \forall \omega \quad \text { in } H_{0}^{1}(\Omega) \\
u(0) & =u_{0} \text { in } \mathrm{H}_{0}^{1}(\Omega), \quad \mathrm{u}_{\mathrm{t}}(0)=\mathrm{u}_{1} \text { in } \mathrm{L}^{2}(\Omega)
\end{aligned}
$$

Corollary 2.1 Assume that the conditions of theorem 2.1 are fulfilled for every $T>0$. Then $u$ is a global weak solution of problem (1.1)-(1.3).

\section{The 1-D case}

In this part we suppose $n=1$ and give additional properties of solutions that we are not able to prove if $n \geq 2$. Indeed, we use the Sobolev inclusion $H_{0}^{1}(\Omega) \subset \mathcal{C}_{0}(\Omega)$ (space of bounded continuous functions) which is valid only if the space dimension is 1 . The main result is a uniqueness result which is a direct consequence of a "local stability" result, namely the continuous dependence of weak solutions on the initial data.

Theorem 3.1 Assume that $n=1$ and that the assumptions of Theorem 2.1 are satisfied for some $T>0$. Let $u, v$ be two solutions of problem (1.1)-(1.3) corresponding (respectively) to initial data $\left(u_{0}, u_{1}\right)$ and $\left(v_{0}, v_{1}\right) \in H_{0}^{1}(\Omega) \times$ $H_{0}^{1}(\Omega)$. Then for almost every $t \in[0, T)$,

$$
\left\|u_{t}(t)-v_{t}(t)\right\|^{2}+\|u(t)-v(t)\|_{1}^{2} \leq M(T)\left(\left\|u_{1}-v_{1}\right\|^{2}+\left\|u_{0}-v_{0}\right\|_{1}^{2}\right),
$$

where $M(T)$ is a positive constant depending on $T$.

Proof - Let $u$ and $v$ be two solutions of problem (1.1)-(1.3) corresponding to initial data $\left(u_{0}, u_{1}\right)$ and $\left(v_{0}, v_{1}\right)$ respectively. We set $\omega=u-v$ and $\omega_{i}=$ $u_{i}-v_{i}, i=0,1$. Then, for every $\varphi \in H_{0}^{1}(\Omega)$ we get

$$
\begin{aligned}
\left(\omega_{t t}, \varphi\right)+(\nabla \omega, \nabla \varphi) & =\left(a(u, p) u_{t}-a(v, p) v_{t}+b\left(u, p, p_{t}\right)-b\left(v, p, p_{t}\right), \varphi\right) \\
\omega & =0 \text { on }[0, \mathrm{~T}) \times \partial \Omega \\
\omega(0, x) & =\omega_{0}, \quad \omega_{t}(0, x)=\omega_{1} \text { in } \Omega
\end{aligned}
$$

Replacing $\varphi$ by $2 \omega_{t}\left(u_{t}\right.$ and $\left.v_{t} \in H_{0}^{1}(\Omega)\right)$ gives

$$
2\left(\omega_{t t}, \omega_{t}\right)+2\left(\nabla \omega, \nabla \omega_{t}\right)=\left(a(u, p) u_{t}-a(v, p) v_{t}+b\left(u, p, p_{t}\right)-b\left(v, p, p_{t}\right), 2 \omega_{t}\right)
$$


and

$$
\begin{gathered}
\frac{d}{d t}\left(\left\|\omega_{t}(t)\right\|^{2}+\|\nabla \omega(t)\|^{2}\right)= \\
\left(\frac{\partial\left(a(u, p) u_{t}\right)}{\partial u}(\beta) \omega+\frac{\partial\left(a(u, p) u_{t}\right)}{\partial u_{t}}(\beta) \omega_{t}, 2 \omega_{t}\right)+\left(\frac{\partial b}{\partial u}(\gamma) \omega, 2 \omega_{t}\right),
\end{gathered}
$$

where $\beta(t, x) \mapsto\left(\beta_{1}(t, x), \beta_{2}(t, x), p(t, x)\right)$ and $\gamma$ are vector functions between $\left(u, u_{t}, p\right),\left(v, v_{t}, p\right)$ and $\left(u, p, p_{t}\right),\left(v, p, p_{t}\right)$ respectively. By $\left(A_{1}\right),\left(A_{2}\right)$ and the Cauchy-Schwartz inequality,

$$
\begin{gathered}
\left.\frac{d}{d t}\left(\left\|\omega_{t}(t)\right\|^{2}+\|\omega(t)\|_{1}^{2}\right) \leq C\left(\left\|\omega_{t}(t)\right\|^{2}+\left\|\omega_{t}(t)\right\|\|\omega(t)\|\right)+\mid\left(\beta_{2} \omega, 2 \omega_{t}\right)\right) \mid \\
\leq C\left(\left\|\omega_{t}(t)\right\|^{2}+\left\|\omega_{t}(t)\right\|\|\omega(t)\|+\left\|\beta_{2}(t)\right\|\left\|\omega_{t}(t)\right\|\|\omega(t)\|_{\infty}\right) .
\end{gathered}
$$

On the other hand, as in the proof of (2.10) we have

$$
\left\|u_{t}(t)\right\|_{1} \text { and }\left\|v_{t}(t)\right\|_{1} \leq M \text { a.e. on }[0, \mathrm{~T}) \text {. }
$$

Therefore, as $\beta_{2}(t, x)$ is between $u_{t}(t, x)$ and $v_{t}(t, x),\left\|\beta_{2}(t)\right\| \leq M$.

Using the Sobolev embedding theorem [1], we get

$$
\|\omega(t)\|_{\infty} \leq C\|\omega(t)\|_{1}
$$

since we have supposed $n=1$. So

$$
\frac{d}{d t}\left(\left\|\omega_{t}(t)\right\|^{2}+\|\omega(t)\|_{1}^{2}\right) \leq M\left(\left\|\omega_{t}(t)\right\|^{2}+\|\omega(t)\|_{1}^{2}\right), \quad \text { a.e. } \mathrm{t} \in[0, \mathrm{~T})
$$

Applying the Gronwall inequality to (3.2), we get

$$
\left\|\omega_{t}(t)\right\|^{2}+\|\omega(t)\|_{1}^{2} \leq M(T)\left(\left\|\omega_{t}(0, x)\right\|^{2}+\|\omega(0, x)\|_{1}^{2}\right) \quad \text { a.e. } \mathrm{t} \in[0, \mathrm{~T}) .
$$

This achieves the proof.

Corollary 3.1 Assume the assumptions of Theorem 2.1 are fulfilled and $n=$ 1. Then problem (1.1)-(1.3) admits a unique weak solution

$$
u \in H^{2}\left([0, T), L^{2}(\Omega)\right) \cap H^{1}\left([0, T), H_{0}^{1}(\Omega)\right) .
$$

\section{Application to cerebral activity model}

We now quickly present a cerebral activity model stated by Jirsa and Haken [9], which we justify mathematically. This model equation is a particular case of equation (1.1).

The elementary unit of the nervous system is the neuron (Fig. 1), which is 
divided into three basic components [7]: dendrites, cell body and axons. A neuron communicates with others at synapses and there are mainly two kinds of synapses [7]: excitatory or inhibitory types. The information transfer between two neurons $\mathrm{A}$ and $\mathrm{B}$ can be described as follows:

- A pulse (electrical signal) arrives at a dendrite of neuron A (under potential action) and emits neurotransmitters (chemical substances) so that it is transformed into a wave. According to [7], this conversion is a linear operation between neural sheets.

- Then the wave reaches the so-called "trigger zone" (Fig. 1) and is converted to a pulse again. Now the conversion law is sigmoidal .

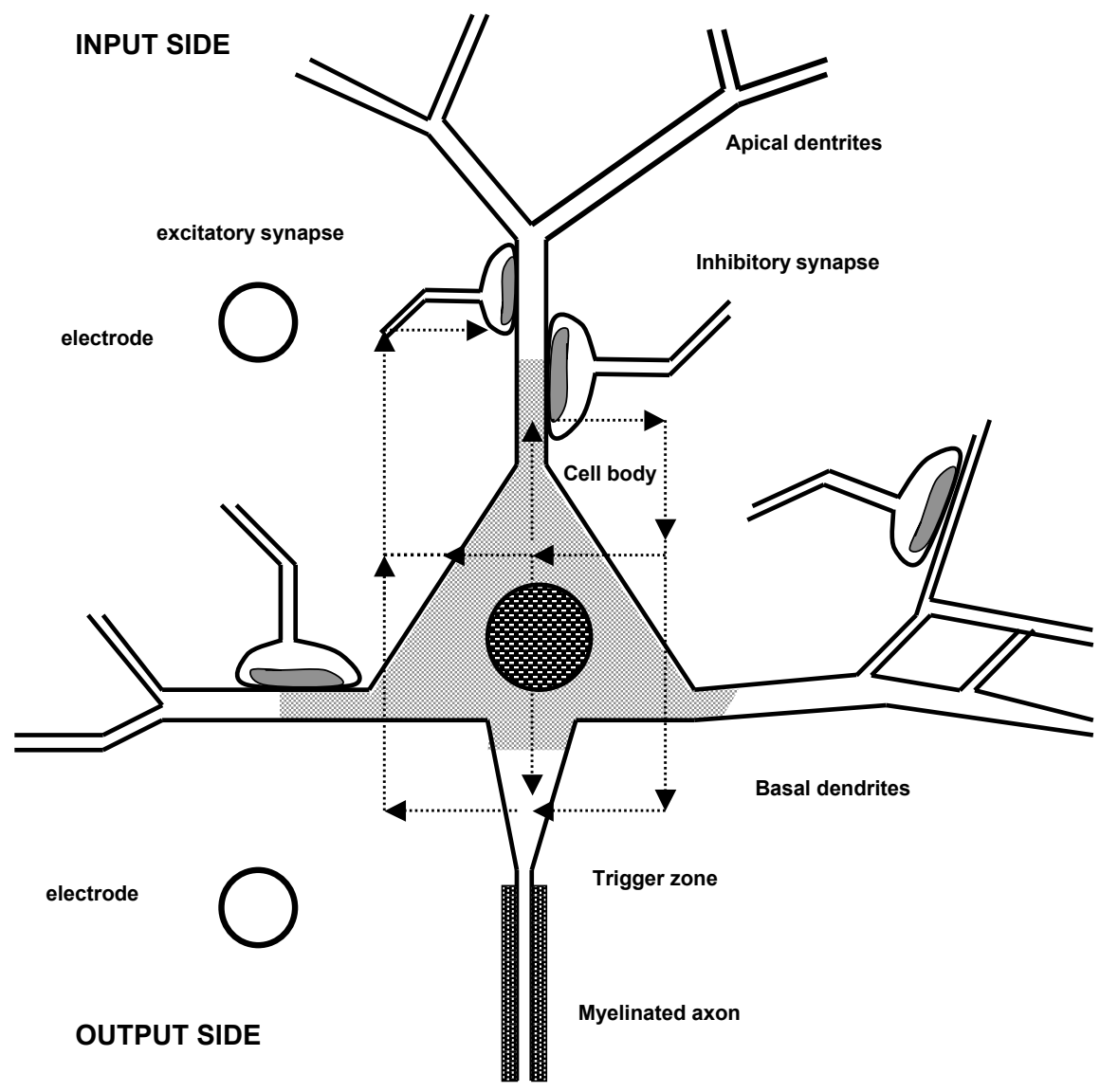

Fig 1. Dendritic trees form thousands of synapses. Excitatory current flows inward at excitatory synapses and outward at the trigger zone. Inhibitory loop current flows in the opposite direction.

The analysis of the conversion operations at the synapses and the trigger zones of neural ensembles allows us to derive a nonlinear partial differential 
field equation describing the spatio-temporal behavior of brain activity.

The pulse and wave variables are classified as two sub-variables according to their excitatory or inhibitory characters: We have the excitatory pulse $E(x, t)$ and inhibitory pulse $I(x, t)$, the excitatory wave $\psi_{e}(x, t)$ and inhibitory wave $\psi_{i}(x, t)$. We can view the wave as

$$
\psi_{k}(x, t)=\int_{\Omega} f_{k}(x, X) H_{k}(x, X, t) d X .
$$

The functions $H_{k}(x, X, t), k=i, e$ are conversion operation outputs and the functions $f_{k}(x, X)$ are the corresponding spatial connectivity distributions. $\Omega$ denotes the neural sheet set (at the brain surface, which is supposed to be continuous). In what follows $\Omega \subset \mathbb{R}$ : we have chosen a 1-D model for the brain geometry as in [8]. We assume that these connectivity functions have the following form:

$$
f_{k} e(x, X)=\frac{1}{2 \sigma_{e}} e^{-\frac{|x-X|}{\sigma_{e}}}
$$

where $k=e, i$ and $\sigma_{e}$ and $\sigma_{i}$ are excitatory and inhibitory connectivity parameters (respectively). Moreover, $\sigma_{i} \ll 1$, since the corticocortical connections (between two distant neurons via corticocortical fibers) are only excitatory. On the other hand, the information propagation along corticocortical fibers implies a delay $t=\frac{|x-X|}{v_{j}}$, where $v_{e}$ and $v_{i}$ are the excitatory and inhibitory propagation speed, respectively, so that we obtain

$$
H_{k}(x, X, t)=a_{k} E\left(X, t-\frac{|x-X|}{v_{k}}\right)
$$

where $k=e, i$ and $a_{e}$ and $a_{i}$ are real constants. Substituting equations (4.3) into (4.1), we obtain:

$$
\begin{aligned}
\psi_{e}(x, t) & =a_{e} \int_{\Omega} f_{e}(x, X) E\left(X, t-\frac{|x-X|}{v}\right) d X \\
\psi_{i}(x, t) & =\int_{\Omega} f_{i}(x, X) H_{i}(x, X, t) d X \\
& \left.\approx f_{i}(x, x) H_{i}(x, x, t)=a_{i} I(x, t) \quad \text { (because } \sigma_{i} \ll 1\right)
\end{aligned}
$$

The pulse value is calculated from the wave value and the external input in the neural tissue (Fig 1); it has a sigmoidal form:

$E(x, t)=S_{e}\left[\psi_{e}(x, t)-\psi_{i}(x, t)+p_{e}(x, t)\right], I(x, t)=S_{i}\left[\psi_{e}(x, t)-\psi_{i}(x, t)+p_{i}(x, t)\right]$

where $p_{k}(x, t)$ is the external input. The sigmoid function $S_{j}$ is the following:

$$
S_{j}\left(n_{j}\right)=\frac{1}{1+\exp \left(-v_{j} n_{j}\right)}-\frac{1}{2}
$$


When $n_{j}$ is small enough, we have a third order approximation of $S_{j}$ :

$$
S_{j}\left(n_{j}\right) \approx n_{j}-\frac{v_{j}^{3} n_{j}^{3}}{48}
$$

Because $\frac{v_{e}}{v_{i}} \approx 100$, we take only the linear part of (4.7) into consideration to obtain:

$$
\psi_{i}(x, t) \approx \frac{a_{i} v_{i}}{4+a_{i} v_{i}}\left(\psi_{e}(x, t)+p_{i}(x, t)\right)
$$

We finally obtain the following equation for the dynamics of the excitatory synaptic activity:

$$
\psi_{e}(x, t)=a_{e} \int_{\Omega} f_{e}(x, X) \cdot S_{e}\left[\widetilde{\rho} \psi_{e}\left(X, t-\frac{|x-X|}{v}\right)+p\left(X, t-\frac{|x-X|}{v}\right)\right] d X
$$

where $p(x, t)=p_{e}(x, t)-\frac{a_{i} v_{i}}{4+a_{i} v_{i}} \cdot p_{i}(x, t)$, and $\widetilde{\rho}=1-\frac{a_{i} v_{i}}{4+a_{i} v_{i}}$.

We suppose hereafter that $\Omega:=(0, L)$ is one-dimensional; that is, we connect all neuron sheets by a line (see [8]), $\psi_{e}(0, t)=\psi_{e}(L, t)=0$ as in [8], and $f_{e}(x, X)$ is an exponential function depending only on the distance between two neural sheets. Therefore

$$
\psi_{e}(x, t)=a_{e} \int_{\Omega} f_{e}(x, X) \cdot S_{e}\left[\widetilde{\rho} \psi_{e}\left(X, t-\frac{|x-X|}{v}\right)+p\left(X, t-\frac{|x-X|}{v}\right)\right] d X
$$

Let us mathematicaly justify the computation of [9].

We set $\rho(X, T)=a_{e} S_{e}(\tilde{\rho} \psi(X, T)+p(X, T))$ and

$$
\begin{aligned}
\mu: L^{1}(\mathbb{R} \times \mathbb{R}) & \rightarrow \mathbb{R} \\
\varphi & \mapsto<\mu, \varphi>=\int_{\mathbb{R}} h(x) \varphi\left(x, \frac{|x|}{v}\right) d x
\end{aligned}
$$

with $h(x)=1_{(0, L)} e^{-\frac{|x|}{\sigma}}$ and $1_{(0, L)}$ the indicator function of $(0, L)$. Then

$$
\begin{aligned}
\mu * \rho(x, t) & =\int_{\mathbb{R}} h(X) g\left(X, \frac{|X|}{v}\right) d X \quad \text { with } g(X, T)=\rho(x-X, t-T) \\
& =\int_{\mathbb{R}} h(X) \rho\left(x-X, t-\frac{|x|}{v}\right) d X \\
& =\int_{\Omega} f_{e}(x, X) \rho\left(X, t-\frac{|x-X|}{v}\right) d X
\end{aligned}
$$

and

$$
\mu * \rho(x, t)=\psi_{e}(x, t) .
$$

The extended Fourier transform gives: $\widehat{\psi_{e}}=\widehat{\mu} \widehat{\rho}$. 
Now, we prove that $\widehat{\mu}=T_{f}, f \in L_{l o c}^{1}(\mathbb{R} \times \mathbb{R})$. Let $\varphi \in \mathcal{D}(\mathbb{R} \times \mathbb{R})$.

$$
\begin{aligned}
<\widehat{\mu}, \varphi>=<\mu, \widehat{\varphi}> & =\int_{\mathbb{R}} h(X) \widehat{\varphi}\left(X, \frac{|X|}{v}\right) d X \\
& =\int_{\mathbb{R}} h(X)\left(\int_{\mathbb{R}} \int_{\mathbb{R}} \varphi(x, t) e^{-2 i \pi\left(x X+t \frac{|X|}{v}\right)} d x d t\right) d X \\
& =\int_{\mathbb{R}} \int_{\mathbb{R}}\left(\int_{\mathbb{R}} h(X) e^{-2 i \pi\left(x X+t \frac{|X|}{v}\right)} d X\right) \varphi(x, t) d x d t \\
& =\int_{\mathbb{R}} \int_{\mathbb{R}} f(x, t) \varphi(x, t) d x d t
\end{aligned}
$$

with $f(x, t)=\frac{1}{2 \sigma_{e}} \int_{\Omega} e^{-\frac{|X|}{\sigma}} e^{-2 i \pi\left(x X+t \frac{|X|}{v}\right)} d X$.

Then

$$
\widehat{\psi_{e}}(x, t)=f(x, t) \widehat{\rho}(x, t) \quad \forall(x, t) \in \mathbb{R} \times \mathbb{R} .
$$

As

$$
\int_{0}^{+\infty} e^{-(a+i \alpha) \zeta} d \zeta=\frac{1}{a+i \alpha}
$$

supposing $\omega_{0}=\frac{v}{\sigma}$, we obtain

$$
\begin{aligned}
f(x, t) & =\frac{1}{2}\left[\frac{1}{\frac{1}{\sigma_{e}}+i\left(\frac{t}{v}-x\right)}+\frac{1}{\frac{1}{\sigma_{e}}+i\left(\frac{t}{v}+x\right)}\right] \\
& =\frac{1}{2}\left[\frac{\omega_{0}}{\omega_{0}+i(t-x v)}+\frac{\omega_{0}}{\omega_{0}+i(t+x v)}\right] \\
& =\left[\frac{\omega_{0}^{2}+i \omega_{0} t}{\left(\omega_{0}+i t\right)^{2}+x^{2} v^{2}}\right] \in L_{l o c}^{1}(\mathbb{R} \times \mathbb{R}) .
\end{aligned}
$$

Equation (4.9) becomes

$$
\widehat{\psi_{e}}(x, t)=\left[\frac{\omega_{0}^{2}+i \omega_{0} t}{\left(\omega_{0}+i t\right)^{2}+x^{2} v^{2}}\right] \widehat{\rho}(x, t) .
$$

Developing equation (4.11), we obtain

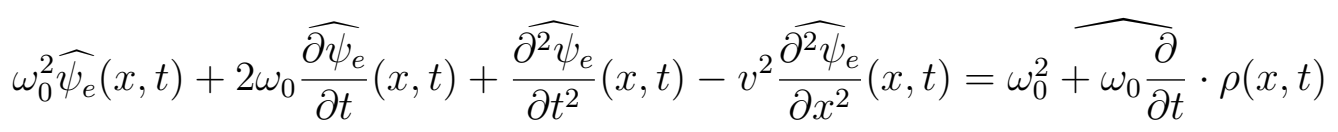

Applying the inverse Fourier Transform, we formally obtain the following partial differential equation:

$$
\frac{\partial^{2} \psi_{e}}{\partial t^{2}}+\left(\omega_{0}^{2}-v^{2} \frac{\partial^{2}}{\partial x^{2}}\right) \psi_{e}+2 \omega_{0} \frac{\partial \psi_{e}}{\partial t}=\left(\omega_{0}^{2}+\omega_{0} \frac{\partial}{\partial t}\right) \cdot \rho
$$

with

$$
\rho(x, t)=a_{e} S_{e}\left(\widetilde{\rho} \psi_{e}(x, t)+p(x, t)\right) .
$$


Developing (4.12) and summing the similar terms gives

$$
\begin{aligned}
\frac{\partial^{2} \psi_{e}}{\partial t^{2}}-v^{2} \frac{\partial^{2} \psi_{e}}{\partial x^{2}} & =\left(a_{e} \omega_{0} \widetilde{\rho} S_{e}^{\prime}\left(\widetilde{\rho} \psi_{e}+p\right)-2 \omega_{0}\right) \frac{\partial \psi_{e}}{\partial t} \\
& -\omega_{0}^{2} \psi_{e}+a_{e} \omega_{0}^{2} S_{e}\left(\widetilde{\rho} \psi_{e}+p\right)+a_{e} \omega_{0} S_{e}^{\prime}\left(\widetilde{\rho} \psi_{e}+p\right) p_{t}
\end{aligned}
$$

We complete this equation with $\psi_{e}(t, 0)=\psi_{e}(t, L)=0$ and Cauchy initial condition. Thanks to the previous sections' results, we have the following:

Theorem 4.1 Assume $p \in H^{2}\left([0, T), L^{\infty}(0, L)\right)$. Then equation (4.13) with $\psi_{e}(t, 0)=\psi_{e}(t, L)=0$ and Cauchy initial condition, with $\left(\psi_{e, 0}, \psi_{e, 1}\right) \in H_{0}^{1}(0, L) \times$ $L^{2}(0, L)$, has a unique weak solution in $H^{2}\left([0, T), L^{2}(0, L)\right) \cap H^{1}\left([0, T), H_{0}^{1}(0, L)\right)$.

Proof - Equation (4.13) is a particular case of equation (1.1), with

$$
a(u, p)=a_{e} \omega_{0} \tilde{\rho} S_{e}^{\prime}(\widetilde{\rho} u+p)-2 \omega_{0}
$$

and

$$
b\left(u, p, p_{t}\right)=-\omega_{0}^{2} u+a_{e} \omega_{0}^{2} S_{e}(\widetilde{\rho} u+p)+a_{e} \omega_{0} S_{e}^{\prime}(\widetilde{\rho} u+p) p_{t} .
$$

If we suppose $p \in H^{2}\left([0, T), L^{\infty}(0, L)\right)$, it is easy to see that functions $a$ and $b$ satisfy the conditions of Theorem 2.1 and consequently, by (2.20) and (3.3), the above equation has a unique weak solution.

\section{Conclusion}

In this paper, we prove the existence of solutions to a new class of wave equations which is motivated by brain activity modeling. In the 1-D spatial dimension case, the solution depends Lipschitz-continuously on the initial data, and so it is unique. This means that the responses to small perturbations are small as well, which is coherent from a physical point of view. We have simplified the model by assuming that:

- the geometry of the brain surface area is 1-D. The 2-D geometry is more consistent but introduces additional difficulties,

- the connectivity functions (4.2) between the neural sheets are homogeneous. In fact more than $50 \%$ of neural sheets have heterogeneous connections, so we should add another term representing the heterogeneity,

- the external input $p$ belongs to $H^{2}\left([0, T), L^{\infty}(\Omega)\right)$. In reality, $p$ may be less smooth or non-local.

In our future work, we will try to generalize our model in order to satisfy the general constraints which are introduced above. 


\section{References}

[1] Robert A. Adams. Sobolev spaces. Academic Press, New York-London, pure and applied mathematics, vol. 65 edition, 1975.

[2] Nikolaos Bournaveas. Low regularity solutions for a class of nonlinear wave equations. Proc. Amer. Math. Soc., 133(9):2721-2727 (electronic), 2005.

[3] J. W. Cholewa and Tomasz Dlotko. Strongly damped wave equation in uniform spaces. Nonlinear Anal., 64(1):174-187, 2006.

[4] Yukiyoshi Ebihara. On some nonlinear evolution equations with the strong dissipation. J. Differential Equations, 30(2):149-164, 1978.

[5] Jorge A. Esquivel-Avila. The dynamics of a nonlinear wave equation. J. Math. Anal. Appl., 279(1):135-150, 2003.

[6] Eduard Feireisl. Global attractors for semilinear damped wave equations with supercritical exponent. J. Differential Equations, 116(2):431-447, 1995.

[7] W.J Freeman. Tutorial on neurobiology: From single neurons to brain chaos. J. Int. J.Bifurcation and chaos, 2:451-482, 1992.

[8] V.K Jirsa. Connectivity and dynamics of neural information processing. neuroinformatics. Neuroinformatics, 2:183-204, 2004.

[9] V.K. Jirsa and H Haken. Field theory of electromagnetic brain activity. Phys, Rev. Let., 77:960-963, 1996.

[10] Tariel Kiguradze. On bounded and time-periodic solutions of nonlinear wave equations. J. Math. Anal. Appl., 259(1):253-276, 2001.

[11] Howard A. Levine, Sang Ro Park, and James Serrin. Global existence and global nonexistence of solutions of the Cauchy problem for a nonlinearly damped wave equation. J. Math. Anal. Appl., 228(1):181-205, 1998.

[12] Howard A. Levine and Grozdena Todorova. Blow up of solutions of the Cauchy problem for a wave equation with nonlinear damping and source terms and positive initial energy. Proc. Amer. Math. Soc., 129(3):793-805 (electronic), 2001.

[13] J.-L. Lions. Quelques méthodes de résolution des problèmes aux limites non linéaires. Dunod, 1969.

[14] Julián López-Gómez. On the linear damped wave equation. J. Differential Equations, 134(1):26-45, 1997.

[15] Kenji Nishihara and Huijiang Zhao. Existence and nonexistence of time-global solutions to damped wave equation on half-line. Nonlinear Anal., 61(6):931-960, 2005 .

[16] P.L. Nunez. The brain wave equation : A model for the eeg. Mathematical Biosciences, 21:279-297, 1974. 
[17] Rafael Orive and Enrique Zuazua. Long-time behavior of solutions to a nonlinear hyperbolic relaxation system. J. Differential Equations, 228(1):17-38, 2006.

[18] Jong Yeoul Park and Jeong Ja Bae. On solutions of quasilinear wave equations with nonlinear damping terms. Czechoslovak Math. J., 50(125)(3):565-585, 2000 .

[19] David R. Pitts and Mohammad A. Rammaha. Global existence and nonexistence theorems for nonlinear wave equations. Indiana Univ. Math. J., 51(6):1479-1509, 2002.

[20] Hervé Queffélec and Claude Zuily. Éléments d'analyse. Dunod, Paris, second edition, 2002.

[21] Grozdena Todorova. Cauchy problem for a nonlinear wave equation with nonlinear damping and source terms. Nonlinear Anal., 41(7-8, Ser. A: Theory Methods):891-905, 2000.

[22] Grozdena Todorova and Borislav Yordanov. Critical exponent for a nonlinear wave equation with damping. C. R. Acad. Sci. Paris Sér. I Math., 330(7):557562,2000 .

[23] H.R. Wilson and J.D. Cowan. Excitatory and inhibitory interactions in localized populations of model neurons. Biophysical Journal, 12:1-24, 1972.

[24] Karen Yagdjian. Global existence in the Cauchy problem for nonlinear wave equations with variable speed of propagation. In New trends in the theory of hyperbolic equations, volume 159 of Oper. Theory Adv. Appl., pages 301-385. Birkhäuser, Basel, 2005.

[25] Qi S. Zhang. A blow-up result for a nonlinear wave equation with damping: the critical case. C. R. Acad. Sci. Paris Sér. I Math., 333(2):109-114, 2001.

[26] Yang Zhijian. Initial boundary value problem for a class of non-linear strongly damped wave equations. Math. Methods Appl. Sci., 26(12):1047-1066, 2003.

[27] Shengfan Zhou and Xiaoming Fan. Kernel sections for non-autonomous strongly damped wave equations. J. Math. Anal. Appl., 275(2):850-869, 2002.

[28] Yong Zhou. A blow-up result for a nonlinear wave equation with damping and vanishing initial energy in $\mathbb{R}^{N}$. Appl. Math. Lett., 18(3):281-286, 2005.

[29] Yong Zhou. Global existence and nonexistence for a nonlinear wave equation with damping and source terms. Math. Nachr., 278(11):1341-1358, 2005. 